\title{
Editorial: Towards the Control of Thermal Expansion: From 1996 to Today
}

\author{
Andrea Sanson ${ }^{1 *}$ and Jun Chen ${ }^{2}$ \\ ${ }^{1}$ Department of Physics and Astronomy, University of Padua, Padua, Italy, ${ }^{2}$ School of Mathematics and Physics, University of \\ Science and Technology Beijing, Beijing, China
}

Keywords: thermal expansion control, negative thermal expansion (NTE), materials physics and chemistry, materials science, solid state physics

\section{Editorial on the Research Topic}

\section{Towards the Control of Thermal Expansion: From 1996 to Today}

Most materials expand on heating, known as positive thermal expansion (PTE). Thermal expansion is critical in many technological applications, like precise instruments, glazes and coating materials, optical and electronic devices, high-temperature materials design. As a matter of fact, different materials in contact with each other can have different thermal expansion, giving rise to thermal shock breakage. For this reason, controlling thermal expansion represents a challenge for materials design.

Negative thermal expansion (NTE), that is material contraction on heating over a certain temperature range, is relatively rare but has important technological applications. Since the large isotropic NTE was discovered in zirconium tungstate over a wide temperature range in 1996, the

\section{OPEN ACCESS}

Edited and reviewed by: Bretislav Friedrich,

Fritz-Haber-Institut, Germany

*Correspondence:

Andrea Sanson

andrea.sanson@unipd.it

Specialty section:

This article was submitted to Physical Chemistry and Chemical

Physics,

a section of the journal

Frontiers in Chemistry

Received: 29 March 2019

Accepted: 08 April 2019

Published: 08 May 2019

Citation:

Sanson A and Chen J (2019) Editorial:

Towards the Control of Thermal

Expansion: From 1996 to Today.

Front. Chem. 7:284.

doi: 10.3389/fchem.2019.00284 interest in NTE has rapidly grown to become the most promising route to achieve the control of thermal expansion. Since then, many NTE materials have been discovered.

NTE phenomenon is known to arise from a range of different physical mechanisms, such as lowenergy vibrational modes, ferroelectricity, magnetic, and valence transitions. However, this does not lead to straightforward control of thermal expansion. Moreover, composites of negative and positive thermal expansion materials may fail after repeated cycling, so direct control of thermal expansion within a single homogenous phase is desirable and yet difficult to achieve. To date, different methods are under investigation to achieve the control of thermal expansion.

The present Research Topic includes a collection of original research and review (mini review) articles dedicated to the physical-chemical phenomena connected to NTE and to the state-of-theart for control thermal expansion. The collection begins with a review by Takenaka on phasetransition type NTE materials, presenting some recent works related to how giant NTE materials are used as practical thermal expansion compensators. Hu et al. present a second review on the NTE behavior in materials with giant magnetocaloric effects (MCE), including some representative examples of MCE materials. An article perspective is presented by Attfield, where NTE is classified in terms of intrinsic structural and electronic mechanisms, and morphological NTE due to the specific morphology of the sample.

Many articles of the collection are dedicated to developing new materials with zero or adjustable thermal expansion. Liu et al. fabricated $\mathrm{Zr}_{2} \mathrm{MoP}_{2} \mathrm{O}_{12} / \mathrm{ZrO}_{2}$ composites with a variable coefficient of thermal expansion (CTE) from $-5.7 \times 10^{-6} \mathrm{~K}^{-1}$ to $+5.6 \times 10^{-6} \mathrm{~K}^{-1}$ by changing the $\mathrm{Zr}_{2} \mathrm{MoP}_{2} \mathrm{O}_{12}$ content, reaching a near zero CTE from 25 to $700^{\circ} \mathrm{C}$ for the composition ratio $\mathrm{Zr}_{2} \mathrm{MoP}_{2} \mathrm{O}_{12} / \mathrm{ZrO}_{2}$ of 2:1. Similarly, Zhang et al. synthetized $\mathrm{Zr}_{2} \mathrm{WP}_{2} \mathrm{O}_{12} / \mathrm{ZrO}_{2}$ composites and adjusted the CTE 
from $-3.3 \times 10^{-6} \mathrm{~K}^{-1}$ to $+4.1 \times 10^{-6} \mathrm{~K}^{-1}$ by varying the mass ratio $\mathrm{Zr}_{2} \mathrm{WP}_{2} \mathrm{O}_{12} / \mathrm{ZrO}_{2}$, obtaining again a zero CTE for the ratio 2:1. Near-zero thermal expansion behavior over a wide temperature range was also found out by $\mathrm{Li}$ et al. in $\mathrm{HfMg}_{1-\mathrm{x}} \mathrm{Zn}_{\mathrm{x}} \mathrm{Mo}_{3} \mathrm{O}_{12}$ solid solutions, whose phase transition temperature from monoclinic to orthorhombic structure increases with the content of $\mathrm{Zn}^{2+}$. Giant NTE properties were observed in $\mathrm{Fe}$-doped $\mathrm{MnNiGe}$ compounds by Zhao et al. with CTE values of about $-285 \times 10^{-6} \mathrm{~K}^{-1}$ and $-1167 \times 10^{-6} \mathrm{~K}^{-1}$ in $\mathrm{Mn}_{0.90} \mathrm{Fe}_{0.10} \mathrm{NiGe}$ and $\mathrm{MnNi}_{0.90} \mathrm{Fe}_{0.10} \mathrm{Ge}$, respectively. Furthermore, these materials were combined with $\mathrm{Cu}$ in order to control their NTE properties. Guo et al. investigated NTE and magnetic properties of anti-perovskite $\mathrm{Ga}_{1-\mathrm{x}} \mathrm{Cr}_{\mathrm{x}} \mathrm{N}_{0.83} \mathrm{Mn}_{3}$ compounds. They observed that $\mathrm{Cr}$ doping introduces ferromagnetic order thus giving rise to the broadening of NTE temperature window. Finally, Zhou et al. obtained huge PTE and NTE in fence-like metal organic frameworks obtained with a series of transition metal ions.

Part of the published works have a computational character. By using an efficient approach for obtaining the variation of the dynamical matrix as a function of the lattice constant, Vila et al. obtained that the large NTE of $\mathrm{ZrW}_{2} \mathrm{O}_{8}$ arises almost exclusively from the transverse contributions of the O-atoms. A second study was performed by Vila et al. on $\mathrm{ZrW}_{2} \mathrm{O}_{8}$, combining theoretical calculations of the structure and vibrational properties with the experimental results from extended x-ray absorption fine structure spectroscopy. Ab initio lattice dynamics calculations were combined with inelastic neutron scattering by Singh et al. to study the NTE dynamics in the metal organic framework compound $\mathrm{AgC}_{4} \mathrm{~N}_{3}$. Ablitt et al. performed density functional theory simulations in RuddlesdenPopper layered perovskite series, showing that by changing the fraction of layer interface in the structure one may control the anisotropic compliance necessary for the pronounced uniaxial NTE observed in these systems. Occhialini et al. discussed the broad issue of structural NTE with particular attention on perovskite-structured materials, providing a model treatment beyond mean field theory to identify the key elements that move toward control of NTE. First-principles calculations were performed in NiSi and isostructural compound NiGe by Goel et al. with the aim of studying the phonon modes which give the major contribution to the NTE behavior in NiSi, and reasons for negligible NTE in NiGe.

Lastly, high-pressure synchrotron powder X-ray diffraction studies of $\mathrm{Cr}_{2} \mathrm{Mo}_{3} \mathrm{O}_{12}$ and $\mathrm{Y}_{2} \mathrm{Mo}_{3} \mathrm{O}_{12}$ were conducted by Young et al. revealing that the monoclinic polymorphs of these materials do not undergo phase transitions within the studied pressure range, making them unique among the $\mathrm{A}_{2} \mathrm{M}_{3} \mathrm{O}_{12}$ family of NTE materials. Jiang et al. investigated the thermal expansion properties of the ultraviolet nonlinear optical crystal $\mathrm{BaAlBO}_{3} \mathrm{~F}_{2}$ and the practical implications were discussed. Wang et al. proposed the new strain gauge method to measure the thermal expansion and magnetostriction of solid materials at low temperatures, opening up an interesting way for exploring potential magnetostrictive and NTE materials.

In summary, the present papers taken together give an overview of research on NTE and on the tuning of thermal expansion. We hope that the different perspectives here reported will inspire new studies and findings toward the design of materials with controlled thermal expansion.

\section{AUTHOR CONTRIBUTIONS}

All authors listed have made a substantial, direct and intellectual contribution to the work, and approved it for publication.

Conflict of Interest Statement: The authors declare that the research was conducted in the absence of any commercial or financial relationships that could be construed as a potential conflict of interest.

Copyright (c) 2019 Sanson and Chen. This is an open-access article distributed under the terms of the Creative Commons Attribution License (CC BY). The use, distribution or reproduction in other forums is permitted, provided the original author(s) and the copyright owner(s) are credited and that the original publication in this journal is cited, in accordance with accepted academic practice. No use, distribution or reproduction is permitted which does not comply with these terms. 